\title{
Contribution to the Study of Ir Electrodes in Aqueous Solution of $\mathrm{KNO}_{3} / \mathrm{HNO}_{3}$ and $\mathrm{KNO}_{3} / \mathrm{HNO}_{3} / \mathrm{Hg}_{2}\left(\mathrm{NO}_{3}\right)_{2}$
}

\author{
E. Milaré, E. Y. Ionashiro, A. V. Benedetti", F. L. Fertonani \\ Instituto de Química, Universidade Estadual Paulista, Araraquara, São Paulo, \\ C.P. 355, CEP 14.801-970, Brazil
}

Received 9 October 2002; accepted in revised form 21 February 2003

\begin{abstract}
Cyclic voltammetry and surface analysis techniques (EDX microanalysis, mapping of elements and SEM images) have been used to study the solid state reactions of Ir in blank solution or $\mathrm{Hg}(\mathrm{I})$-containing solution. The studies in $\mathrm{KNO}_{3} / \mathrm{HNO}_{3}$ solution permit to suggest the formation of a thin oxide layer film deposited in the substrate. The RCV process exhibits the decrease of capacitive current associated to the substrate surface restructuring. The electro deposition of $\mathrm{Hg}$ occurs initially in UPD condition from $\mathrm{Hg}(\mathrm{I})$-containing solution, $\mathrm{C}_{\mathrm{Hg}(\mathrm{I})}=3.51 \times 10^{-7} \mathrm{~mol} \mathrm{~L}^{-1}$. The electro deposition of bulk mercury occurs from $\mathrm{Hg}(\mathrm{I})$-containing solution, $\mathrm{C}_{\mathrm{Hg}(\mathrm{I})}=3.51 \times 10^{-5} \mathrm{~mol} \mathrm{~L}^{-1}$. It was observed the deposition of $\mathrm{Hg}$ in the substrate in open circuit for all employed conditions.
\end{abstract}

Keywords: iridium, mercury, cyclic voltammetry, UPD.

\section{Introdução}

Em trabalhos recentes os metais Ir, Rh, Pt e ligas destes metais Pt-Ir e Pt-Rh têm sido empregados como catalisadores suportados em alumina para $\mathrm{o}$ craqueamento do petróleo, na indústria petroquímica [1], suportados sobre $\mathrm{SiC}$, para catálise [2]; em $\mathrm{SiO}_{2}$ e $\mathrm{TiO}_{2}$ para a oxidação exaustiva do $\mathrm{NO}$ para $\mathrm{NO}_{2} \mathrm{e}$ $\mathrm{SO}_{2}$ para $\mathrm{SO}_{3}[3,4]$; como catalisador na oxidação da amônia em plantas industriais [5], em contatos elétricos [6], e no preparo de macro e microeletrodos em eletroquímica e eletroanalítica [7-9].

\footnotetext{
* Corresponding author. E-mail address: benedeti@iq.unesp.br. Communication presented at XV IberoAmerican Meeting of Electrochemistry.
} 
Trabalho recente desenvolvido por Arvia et al. [10] mostrou que a formação e redução de filmes de óxidos na superfície do substrato de Ir ocorre de maneira distinta dos outros metais do grupo da Pt. No Ir a transição da monocamada ou submonocamada do óxido para as camadas de óxido hidratado é seguida por um aumento progressivo na sua espessura com o aumento do potencial, que depende muito do potencial de inversão. A presença de tais óxidos pode contribuir para a penetração do Hg na rede cristalina do Ir e aumentar a reatividade do substrato frente ao $\mathrm{Hg}$ eletrodepositado.

Um outro fator importante a ser considerado é a ação corrosiva do $\mathrm{Hg}$ sobre os metais puros e ligas suportadas. A elevada reatividade do $\mathrm{Hg}$ tem um caráter ambíguo, podendo ser benéfico ou não, dependendo da aplicação do material resultante. No caso do craqueamento do petróleo, o $\mathrm{Hg}$ presente como contaminante, reage com o substrato metálico do catalisador formando compostos intermetálicos com o catalisador.

Desta forma, o presente trabalho tem por objetivo estudar a influência da deposição de filmes finos de óxidos sobre a reatividade do substrato de Ir frente ao $\mathrm{Hg}$ empregando a voltametria cíclica (VC) para a deposição e remoção de mercúrio e técnicas complementares de análise da superfície e dos compostos formados.

\section{Experimental}

Lâminas de Ir puro $\left(80 \mathrm{~mm}^{2}\right.$ ) foram polidas com $\alpha-\mathrm{Al}_{2} \mathrm{O}_{3}$ (tamanho das partículas $<0.3 \mu \mathrm{m})$ em suspensão aquosa e lavadas em $\mathrm{HNO}_{3}: \mathrm{H}_{2} \mathrm{O}(50 \% \mathrm{v} / \mathrm{v})$ em banho de ultra-som. Estas lâminas foram, então, submetidas aos tratamentos em solução de $\mathrm{KNO}_{3} / \mathrm{HNO}_{3}\left(\mathrm{pH}\right.$ 1) ou $\mathrm{KNO}_{3} / \mathrm{HNO}_{3}(\mathrm{pH} 1) / \mathrm{Hg}_{2}\left(\mathrm{NO}_{3}\right)_{2}$.

Todos os dados eletroquímicos foram obtidos empregando-se um potenciostato / galvanostato ECOCHEMIE PGSTAT10 e uma célula eletroquímica que permitia a troca de matriz $(\mathrm{V}=10 \mathrm{~mL})$ com: 1- eletrodo de trabalho - lâmina de Ir (ca. 85 $\left.\mathrm{mm}^{2}\right)$; 2- eletrodo auxiliar - lâmina de $\operatorname{Pt}\left(\mathrm{A}=0.1 \mathrm{~cm}^{2}\right)$; 3- eletrodo de referência

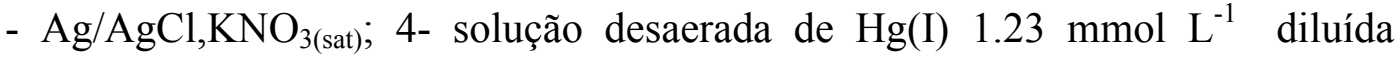
conforme as necessidades e $1.00 \mathrm{M} \mathrm{KNO}_{3} / \mathrm{HNO}_{3}, \mathrm{pH} 1$. Os eletrodos foram 
estudados empregando a técnica da voltametria cíclica repetitiva - VCR (1 000 ciclos a $\mathrm{v}=1 \mathrm{~V} \mathrm{~s}^{-1}$ no intervalo de potenciais $\mathrm{E}_{\mathrm{i}}=\mathrm{E}_{\mathrm{f}}=0.50 \mathrm{~V}, \mathrm{E}_{\lambda, 1}=-0.10 \mathrm{~V}$; $\mathrm{E}_{\lambda, 2}=0.70 \mathrm{~V}$ ) em meio contendo $\mathrm{KNO}_{3} / \mathrm{HNO}_{3}, \mathrm{pH} 1$ (eletrólito de suporte). Após este tratamento os eletrodos foram estudados em solução de eletrólito de suporte nas mesmas condições da VCR e em solução contendo íons $\mathrm{Hg}(\mathrm{I})$, $3.51 \times 10^{-7} \leq \mathrm{C}_{\mathrm{Hg}(\mathrm{I})} \leq 3.51 \times 10^{-5} \mathrm{~mol} \mathrm{~L}^{-1}$, no intervalo de potenciais $\mathrm{E}_{\mathrm{i}}=\mathrm{E}_{\mathrm{f}}=0.50$ $\mathrm{V}, \mathrm{E}_{\lambda, 1}=-0.15 \mathrm{~V} ; \mathrm{E}_{\lambda, 2}=0.80 \mathrm{~V}$.

A superfície das amostras foi estudada empregando-se a microscopia eletrônica de varredura com um microscópio JEOL JSM-T330A acoplado a um sistema NORAN para obtenção dos espectros de EDX antes e após os experimentos.

\section{Resultados e Discussão}

A Fig. 1 mostra os voltamogramas cíclicos obtidos antes e após a VCR em solução de eletrólito de suporte. Nesta figura é possível observar uma diminuição nos valores de corrente após a VCR. Tal diminuição pode ser atribuída a uma reestruturação do substrato conforme sugerem Arvia et al. [11-13] em trabalho com o substrato de Pt, empregando a técnica da voltametria de onda quadrada. É importante notar que sempre há a presença de pelo menos um pequeno pico catódico e um pico anódico, devido à formação e redução de óxido, o que poderia estar modificando a estrutura superficial do Ir. O detalhe na Fig. 1 mostra o voltamograma cíclico já estabilizado, obtido para $\mathrm{v}=1 \mathrm{~V} \mathrm{~s}^{-1}$.

Após a ativação, o voltamograma cíclico em solução de eletrólito de suporte foi obtido empregando-se diferentes velocidades de varredura, Fig. $2(1.25 \leq \mathrm{v} \leq$ $300 \mathrm{mV} \mathrm{s}^{-1}$ ). É importante notar a grande quantidade de ruído, especialmente para as baixas velocidades de varredura, o que dificulta muito os estudos nestas velocidades. Como os ruídos apresentavam sempre as mesmas características e freqüências procurou-se eliminar todas as prováveis fontes, lixando e refazendo os contatos, verificou-se o aterramento do equipamento, etc. Esses procedimentos minimizaram os ruídos mas não os eliminaram completamente.

Nesta figura, é possível verificar que para potenciais próximos a $0.6 \mathrm{~V}$ se obtém e se reduz uma pequena quantidade de óxido. Com a finalidade de verificar se 
haveria qualquer tipo de influência do avanço do potencial sobre a formação / redução de óxidos na região de potenciais de interesse, foram realizados experimentos com inversão de potenciais nas regiões anódica e catódica (Figs. 3a e $b$, respectivamente). Os voltamogramas cíclicos foram obtidos iniciando-se a varredura em $E_{i}=-0.1 \mathrm{~V}$ com inversões a cada $0.05 \mathrm{~V}$ e avanço para regiões mais anódicas até $0.6 \mathrm{~V}$.

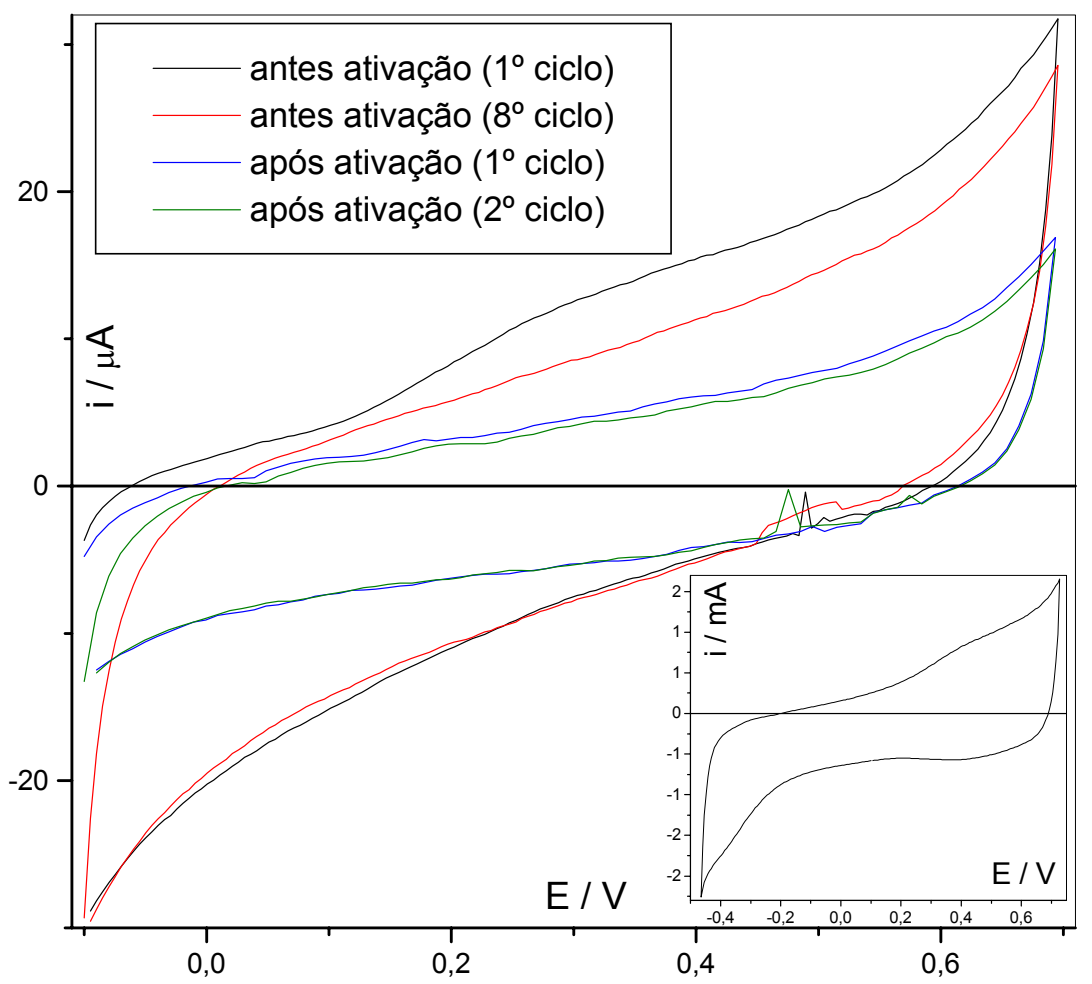

Figura 1. Voltamogramas cíclicos obtidos sobre o eletrodo de Ir puro antes e após a VCR em solução de $\mathrm{KNO}_{3}, \mathrm{C}=1.00 \mathrm{~mol} \mathrm{~L}^{-1}, \mathrm{pH} 1.00\left(\mathrm{HNO}_{3}\right), \mathrm{v}=0.05 \mathrm{~V} \mathrm{~s}^{-1}$. No detalhe, milésimo ciclo obtido a $1 \mathrm{~V} \mathrm{~s}^{-1} . \mathrm{T}=(25 \pm 1)^{\circ} \mathrm{C}$.

Pode-se notar pela Fig. 3a que, mesmo após lavagem e tratamento com ácido nítrico concentrado, na tentativa de eliminar possíveis óxidos da superfície, a formação de óxidos tem início próximo a $0.20 \mathrm{~V}$, demonstrando que este processo pode estar coexistindo com a oxidação / redução de $\mathrm{Hg}$ sobre o substrato. A correlação entre as cargas catódica e anódica para esta figura apresenta valores crescentes a partir de 0.33 para $\mathrm{E}=0.05 \mathrm{~V}$, tendendo à estabilidade a partir de $\mathrm{E}=0.2 \mathrm{~V}$ quando atinge valores próximos a 0.95 (Fig. 4), 
indicando, desta forma, que praticamente todo o óxido formado na varredura anódica está sendo reduzido na varredura catódica.
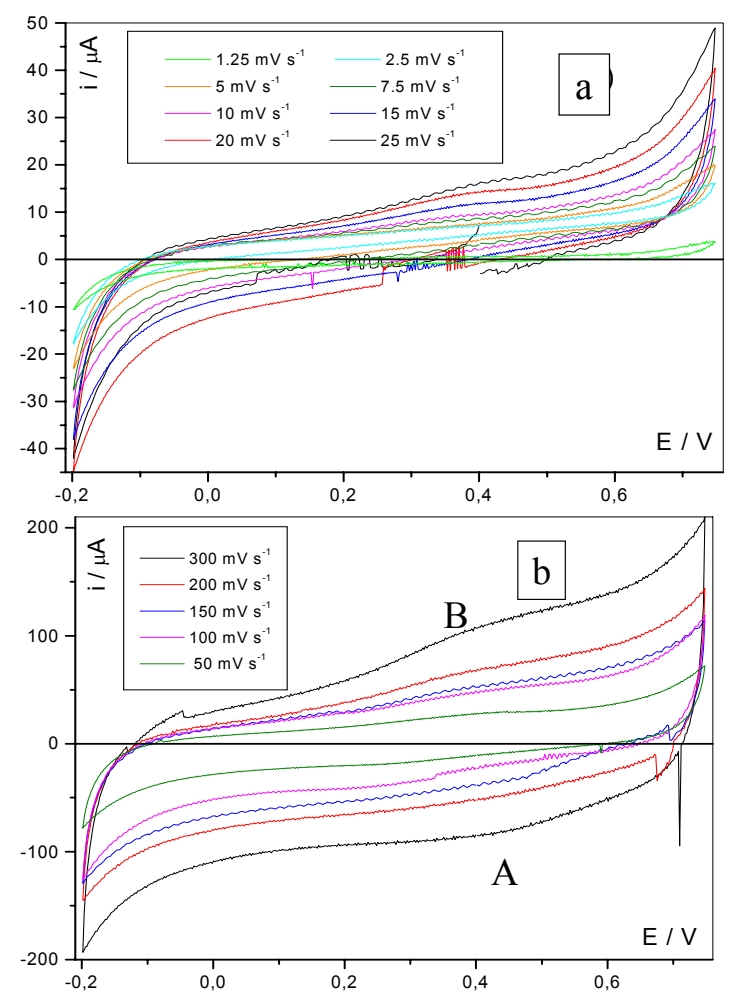

Figura 2. Voltamogramas cíclicos obtidos para o eletrodo de Ir em diferentes valores de $\mathrm{v}$ : $0.00125 \leq \mathrm{v} \leq 0.3 \mathrm{~V} \mathrm{~s}^{-1}$; a) baixos; b) altos valores de v; solução de $\mathrm{KNO}_{3}, \mathrm{C}=1.00$ $\mathrm{mol} \mathrm{L}{ }^{-1}, \mathrm{pH} 1.00 . \mathrm{T}=(25 \pm 1)^{\circ} \mathrm{C}$

É importante notar pelas Figs. 2 (pico A) e 3b que a redução da camada de óxido tem seu início em potenciais próximos a $0.6 \mathrm{~V}$, tendo seu pico em $0.5 \mathrm{~V}$, potencial onde já se inicia o processo de deposição do Hg na condição de UPD sobre o substrato de Ir. Este fato está em concordância com o observado por Arvia et al. [10] que encontraram evidências de que ocorre redução de óxidos até próximo ao potencial de descarga de hidrogênio. Entretanto, a carga desta camada de óxido pode ser desprezada quando comparada à carga de mercúrio. 

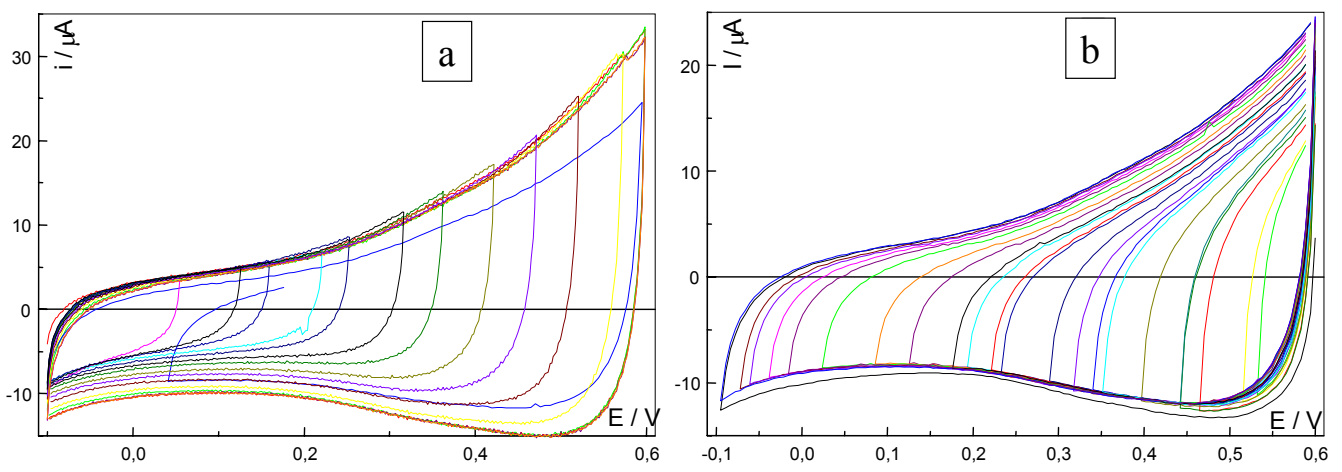

Figura 3. Voltamogramas cíclicos obtidos para o eletrodo de Ir puro, para diferentes valores de potencial de inversão durante a varredura anódica $(\mathrm{A})$ e catódica $(\mathrm{B}) ; \mathrm{v}=$ $0.05 \mathrm{~V} \mathrm{~s}^{-1}$; solução de $\mathrm{KNO}_{3}, \mathrm{C}=1.00 \mathrm{~mol} \mathrm{~L}{ }^{-1}, \mathrm{pH} 1.00\left(\mathrm{HNO}_{3}\right) . \mathrm{T}=(25 \pm 1){ }^{\circ} \mathrm{C}$

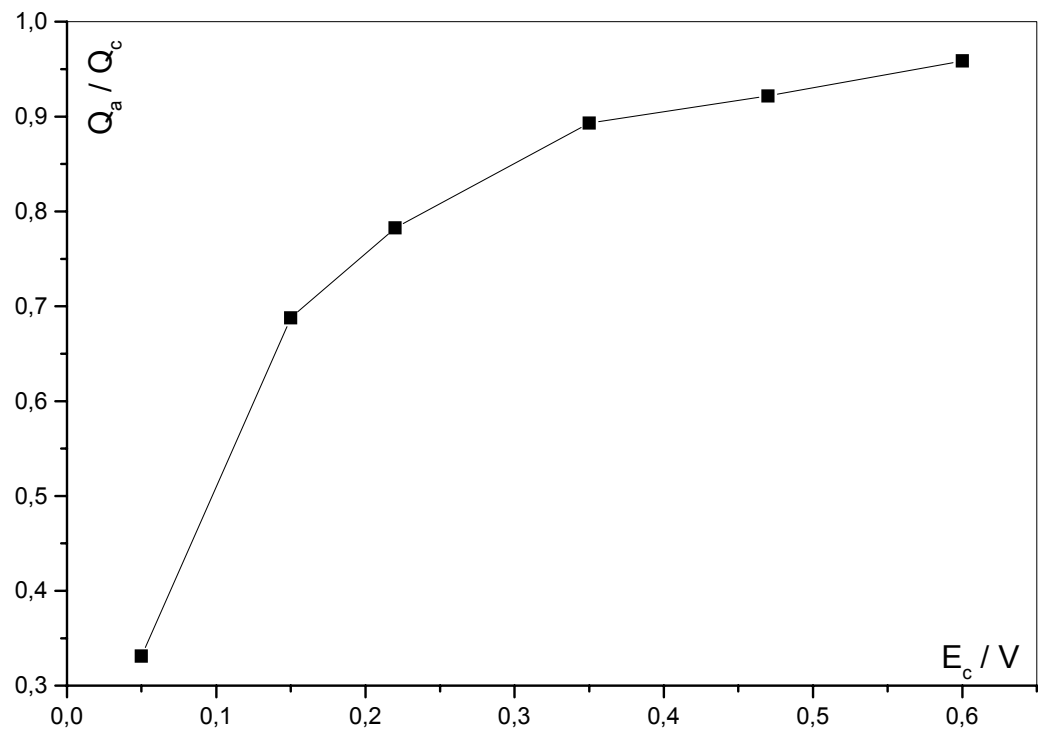

Figura 4. Correlação entre as cargas catódica e anódica obtidas a partir dos voltamogramas cíclicos apresentados na Fig. 3b, para diferentes valores de potencial de inversão durante a varredura anódica; $\mathrm{v}=0.05 \mathrm{~V} \mathrm{~s}^{-1}$; solução de $\mathrm{KNO}_{3}, \mathrm{C}=1.00 \mathrm{~mol}$ $\mathrm{L}^{-1}, \mathrm{pH} 1.00\left(\mathrm{HNO}_{3}\right) . \mathrm{T}=(25 \pm 1){ }^{\circ} \mathrm{C}$

Após a obtenção dos voltamogramas cíclicos apresentados na Fig. 3 (a e $\underline{\mathrm{b}}$ ), procedeu-se a obtenção das imagens SEM, da microanálise por EDX e do mapeamento de elementos, empregando-se a lâmina utilizada nestes estudos e uma lâmina sem contato com as soluções (Figs. 5 e 6). Comparando-se estas amostras é possível verificar em ambas a ausência de contorno de grãos e a presença de um grande número de buracos, conforme já fora observado anteriormente [14]. Na Fig. 5b a amostra encontra-se impregnada por cristais de 
$\mathrm{KNO}_{3}$, conforme pode ser observado a partir do resultado do mapeamento obtido para o irídio e o potássio (Figs. 6a e b, respectivamente).
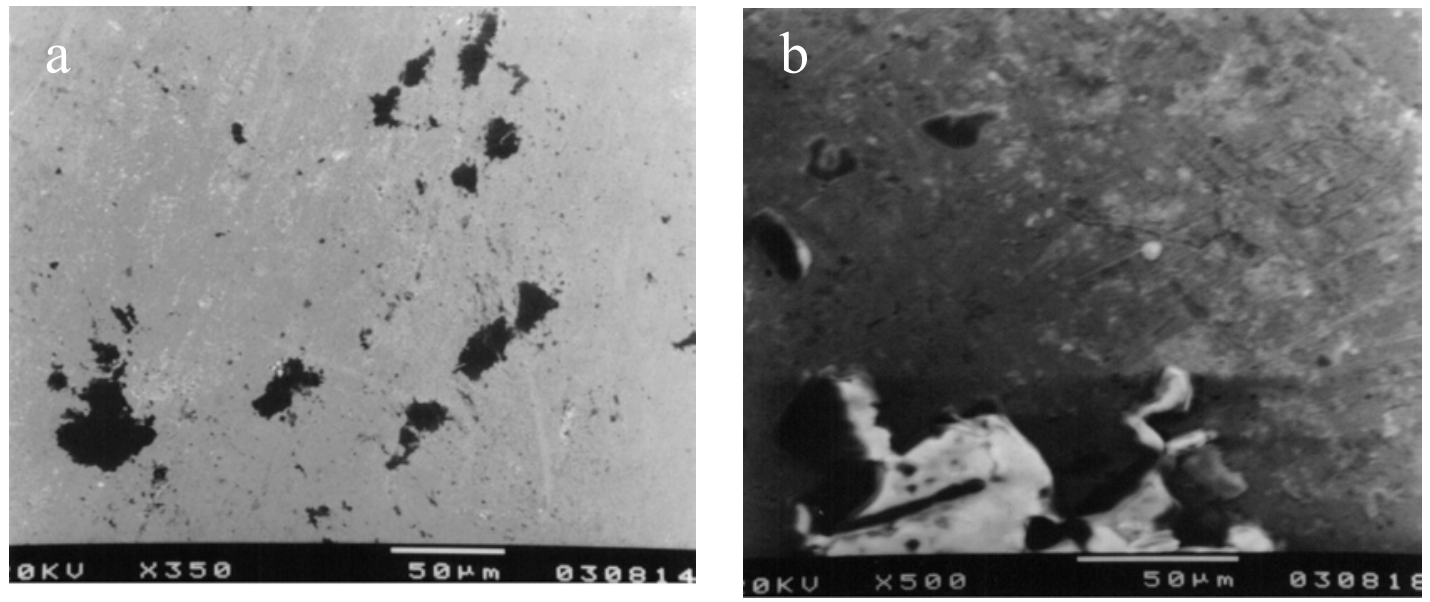

Figura 5. Imagens SEM obtidas para: a) amostra lavada com $\mathrm{HNO}_{3}$ sem contato com a solução do branco, ampliação $=350 \times$; b) amostra posterior à realização dos voltamogramas cíclicos com inversão de potenciais em solução de eletrólito de suporte (Fig. 3). Ampliação $=500 \times$. Energia do feixe: $30 \mathrm{keV}$. Solução de $\mathrm{KNO}_{3}, \mathrm{C}=1.00 \mathrm{~mol}$ $\mathrm{L}^{-1}, \mathrm{pH} 1.00\left(\mathrm{HNO}_{3}\right)$.
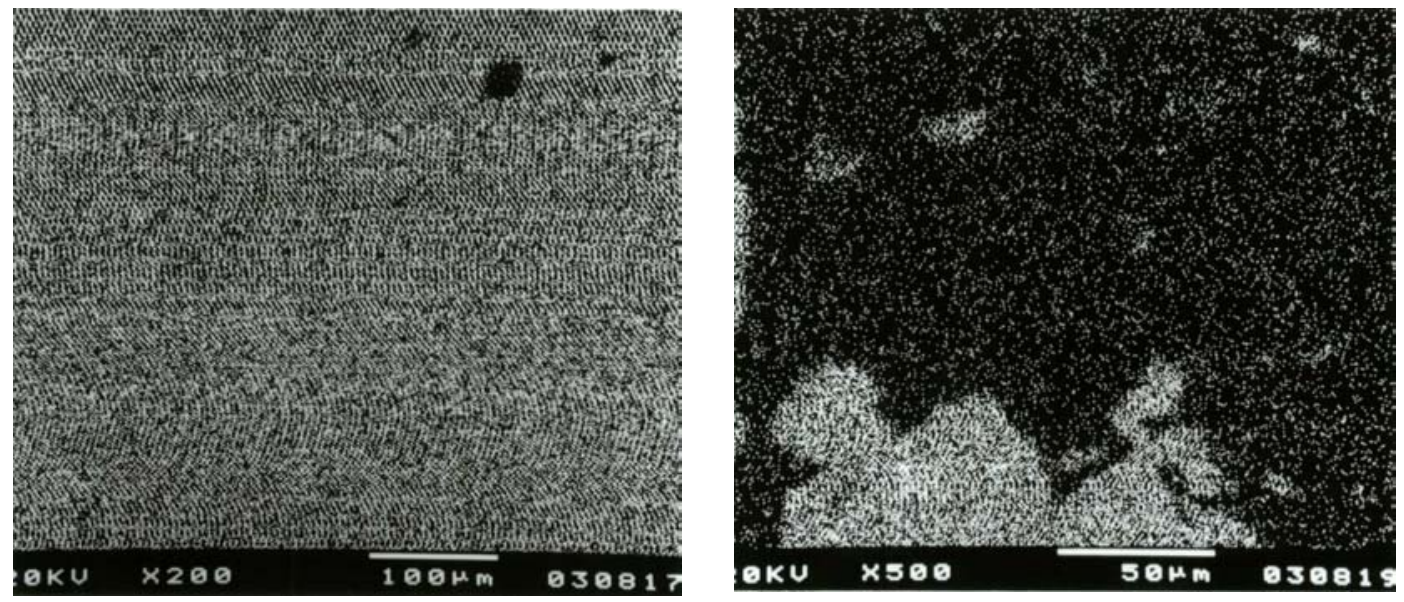

Figura 6. Mapeamento dos elementos Ir e K após obtenção dos voltamogramas cíclicos com inversão de potenciais em solução de eletrólito de suporte (Fig. 3): a) mapeamento do $\mathrm{Ir}$, ampliação $=200 \times$; b) mapeamento do K, ampliação $=500 \times$. Energia do feixe $=$ $30 \mathrm{kV}$. Solução de $\mathrm{KNO}_{3}, \mathrm{C}=1.00 \mathrm{~mol} \mathrm{~L}^{-1}, \mathrm{pH} 1.00\left(\mathrm{HNO}_{3}\right)$.

Estudos prévios mostram que, para os sistemas $\mathrm{Rh}-\mathrm{Hg}$ e Pt-Hg, é possível obter filmes finos de compostos intermetálicos do tipo $\mathrm{RhHg}$ e $\mathrm{PtHg}_{4}$, respectivamente, para concentrações de $\mathrm{Hg}(\mathrm{I})$ em torno de $0.20 \mathrm{mmol} \mathrm{L}^{-1}$ [15-21] na ausência de 
Hg volumétrico, o mesmo não sendo possível para o sistema $\operatorname{Ir}-\mathrm{Hg}[16,21,22]$. Assim sendo, uma das primeiras preocupações no estudo deste sistema foi diminuir a concentração do $\mathrm{Hg}(\mathrm{I})$ de tal modo que pudesse ser obtida apenas uma monocamada de $\mathrm{Hg}$, sem a presença de $\mathrm{Hg}$ volumétrico.

A obtenção do filme fino de $\mathrm{Hg}$ sobre o substrato sem a formação de $\mathrm{Hg}$ volumétrico só foi possível para valores de concentrações entre $3.5 \times 10^{-7} \mathrm{~mol} \mathrm{~L}^{-1}$ e $3.5 \times 10^{-6} \mathrm{~mol} \mathrm{~L}^{-1}$. A Fig. 7 apresenta voltamogramas cíclicos obtidos no intervalo de potenciais $-0.15 \leq \mathrm{E} \leq 0.8 \mathrm{~V}$ e concentrações $3.51 \times 10^{-7}$ (Fig. 7a), $3.51 \times 10^{-6}$ (Fig. 7 b) e $3.51 \times 10^{-5}$ (Fig. 7 c).

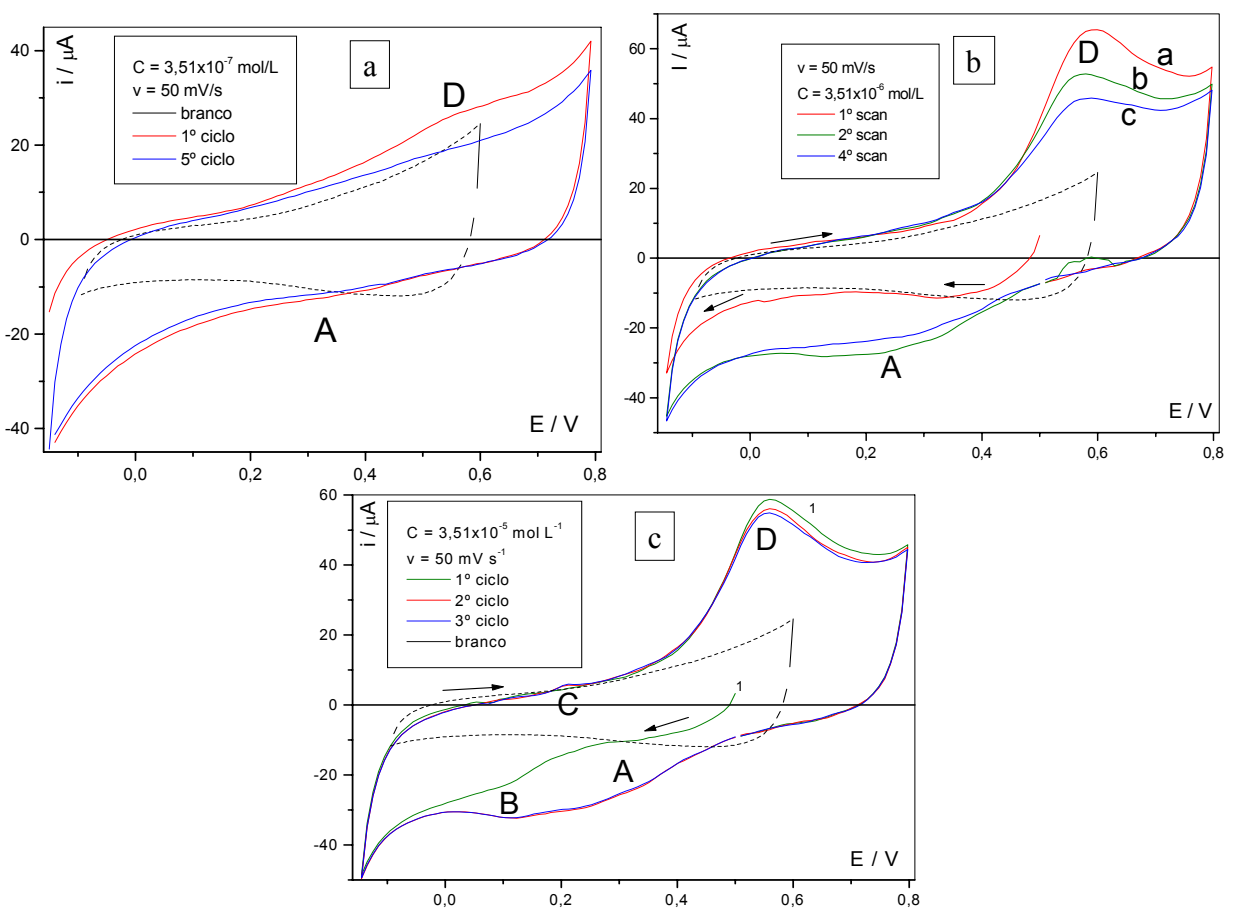

Figura 7. Voltamogramas cíclicos do processo de redução e oxidação de $\mathrm{Hg}$ obtidos para o eletrodo de Ir após 15 minutos de agitação da solução: (a) $\mathrm{C}_{\mathrm{Hg}(\mathrm{I})}=3.51 \times 10^{-7} \mathrm{~mol}$ $\mathrm{L}^{-1}$; (b) $\mathrm{C}_{\mathrm{Hg}(\mathrm{I})}=3.51 \times 10^{-6} \mathrm{~mol} \mathrm{~L}^{-1} ; \mathrm{C}_{\mathrm{Hg}(\mathrm{I})}=3.51 \times 10^{-5} \mathrm{~mol} \mathrm{~L}^{-1} ; \mathrm{v}=0.05 \mathrm{~V} \mathrm{~s}^{-1}$, eletrólito de suporte $\mathrm{KNO}_{3} \mathrm{C}=1 \mathrm{~mol} \mathrm{~L}^{-1}, \mathrm{pH}=1.00 ; \mathrm{T}=(25 \pm 1)^{\circ} \mathrm{C}$

A partir da Fig. 7a é possível observar que a quantidade de $\mathrm{Hg}(\mathrm{I})$ presente em solução é suficiente apenas para formar o filme no primeiro ciclo depois de 15 minutos de agitação da solução com fluxo contínuo de $\mathrm{N}_{2}$. A partir do segundo 
ciclo já não é observada a presença dos picos de deposição de Hg em UPD (pico $\mathrm{A}-0.5 \geq \mathrm{E} \geq 0.2 \mathrm{~V}$ ) e sua posterior oxidação (pico $\mathrm{D}-0.35 \leq \mathrm{E} \leq 0.70 \mathrm{~V}$ ). Para concentração de $\operatorname{Hg}(\mathrm{I})$ em solução dez vezes superior (Fig. 7b) é possível observar que para o primeiro ciclo a carga da UPD está bastante diminuída, isso porque em potencial de circuito aberto $\left(\mathrm{E}_{\mathrm{ca}}=0.31 \mathrm{~V}\right)$ já ocorre a deposição de $\mathrm{Hg}$ sobre o Ir. A referida diminuição está associada ao processo oxidativo observado no início deste ciclo (Fig. 7b, curva 1) removendo, portanto, parte do $\mathrm{Hg}$ resultante da UPD. Esta remoção parcial do Hg depositado em UPD ocorre pelo fato do potencial passar de $0.31 \mathrm{~V}$ (condição de circuito aberto) para $0.50 \mathrm{~V}$, potencial de início da varredura, quase que instantaneamente. A deposição na condição de circuito aberto é relatada na literatura por Machado et al. [23] para $\mathrm{Pt}, \mathrm{Pb}, \mathrm{Zn}$ e $\mathrm{Cd}$, e, neste trabalho, são apresentadas razões para a ocorrência desse processo. Então, supõe-se que o sistema Ir-Hg apresente comportamento similar. Neste trabalho, foram observadas pequenas gotas de mercúrio sobre a superfície do Ir após longos tempos de imersão, em soluções contendo altas concentrações de $\mathrm{Hg}_{2}{ }^{2+}$, em condição de circuito aberto.

A presença dos picos $\mathrm{B}$ (deposição de $\mathrm{Hg}$ volumétrico, $\mathrm{E} \leq 0.2 \mathrm{~V}$ ) e C (oxidação de $\mathrm{Hg}$ volumétrico, $0.18 \leq \mathrm{E} \leq 0.25 \mathrm{~V}$ ) na Fig. 7c indica que a concentração de Hg (I) não é suficientemente baixa para evitar a formação do Hg volumétrico. No entanto, convém destacar o aumento de intensidade dos picos ( $\mathrm{B}$ e $\mathrm{C}$ ) ao passar do primeiro para o segundo ciclo. $\mathrm{O}$ aumento dos referidos picos pode ser explicado analisando-se o processo de UPD. Anterior ao início da varredura, o sistema é deixado por 15 minutos sob fluxo constante de $\mathrm{N}_{2}$. Nesse intervalo de tempo, ocorre a deposição lenta do $\mathrm{Hg}_{2}{ }^{2+}$, na condição de circuito aberto $\left(\mathrm{E}_{\mathrm{OC}}=\right.$ $0.31 \mathrm{~V}$ ), anterior ao registro do primeiro ciclo. Esse tempo pode permitir que ocorra uma maior deposição de $\mathrm{Hg}$ na condição de UPD, provavelmente devido a um melhor arranjo dos átomos de Hg na superfície do Ir. Nesta condição, parece não haver Hg disponível para a formação da fase volume. Posterior à remoção desta monocamada, durante o primeiro ciclo, o Hg é depositado e removido sem o tempo de repouso. Assim, menor quantidade de Hg seria depositada em UPD, sobrando $\mathrm{Hg}_{2}{ }^{2+}$, em solução, suficiente para originar os picos de deposição do $\mathrm{Hg}$ 
em fase volume nos ciclos subseqüentes. Desta forma, é importante observar que o aumento dos picos $\mathrm{B}$ e $\mathrm{C}$, correspondentes ao $\mathrm{Hg}$ em fase volume, ocorre de maneira concomitante à diminuição do pico $\mathrm{D}$, demonstrando que a quantidade de $\mathrm{Hg}$ depositado na condição de UPD, para os voltamogramas cíclicos subseqüentes, é menor do que a quantidade depositada na condição de circuito aberto.

Para verificar a dependência dos picos de UPD (A e D) foram obtidos voltamogramas com a inversão do sentido de varredura em vários potenciais para o ramo catódico (Fig. 8). Nota-se que os picos A e D não se deslocam no eixo de potenciais com a mudança dos potenciais de inversão de varredura.

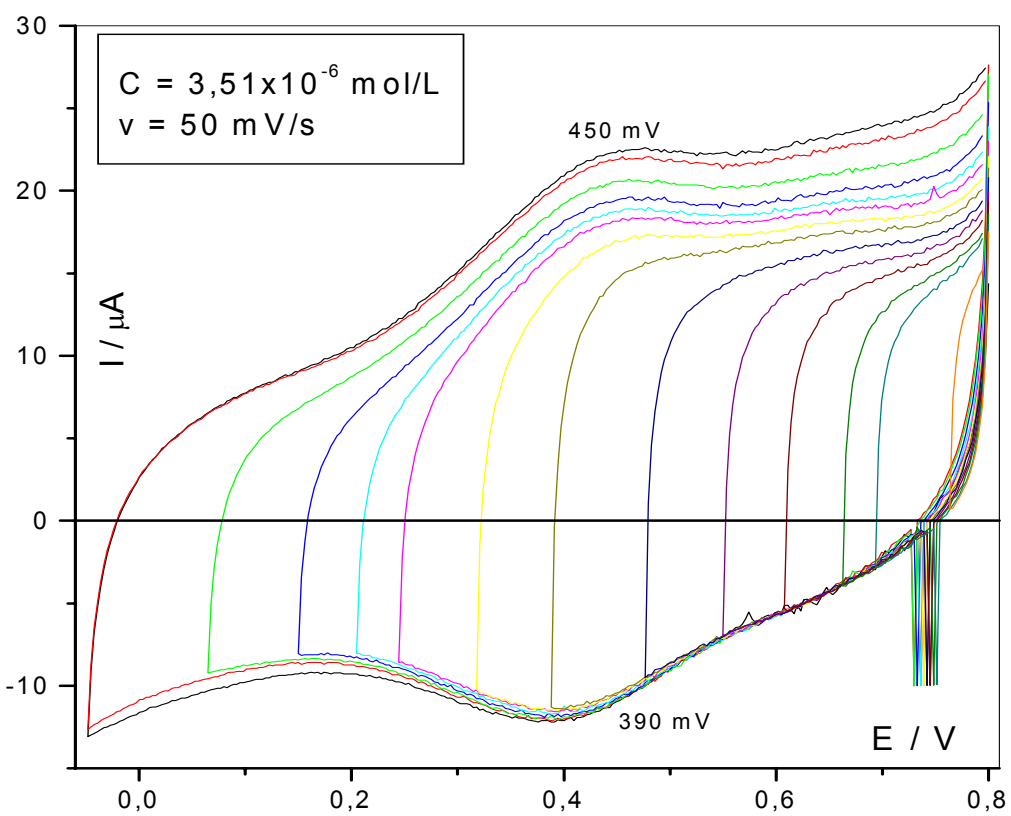

Figura 8. Voltamogramas cíclicos obtidos para o eletrodo de Ir puro com cortes de potencial e subseqüente inversão da varredura para o ramo catódico: $\mathrm{C}_{\mathrm{Hg}(\mathrm{I})}=3.51 \times 10^{-6}$ mol L ${ }^{-1}, \mathrm{v}=0.05 \mathrm{~V} \mathrm{~s}^{-1}$; eletrólito de suporte $\mathrm{KNO}_{3} \mathrm{C}=1 \mathrm{~mol} \mathrm{~L}{ }^{-1} ; \mathrm{pH} 1.00 ; \mathrm{T}=(25 \pm 1)$ ${ }^{\circ} \mathrm{C}$.

A Fig. 9 apresenta as imagens SEM (Figs. 9 $\underline{a}$ e $\underline{b}$ ) e o mapeamento de $\mathrm{Hg}$ (Fig. 9c) obtidos para os eletrodos de Ir preparados empregando $\mathrm{C}_{\mathrm{Hg}(\mathrm{I})}=3.51 \times 10^{-6} \mathrm{~mol}$ $\mathrm{L}^{-1}$. As amostras submetidas à voltametria cíclica para concentrações menores não revelaram a presença de $\mathrm{Hg}$. As imagens SEM revelaram uma superfície 
livre da presença de Hg volumétrico e supostamente não atacada pelo mercúrio, mas com a presença de buracos, como observado anteriormente (Fig. 5), porém desta vez mais intensos, podendo esta intensidade ter sido causada pela ação do $\mathrm{Hg}$.

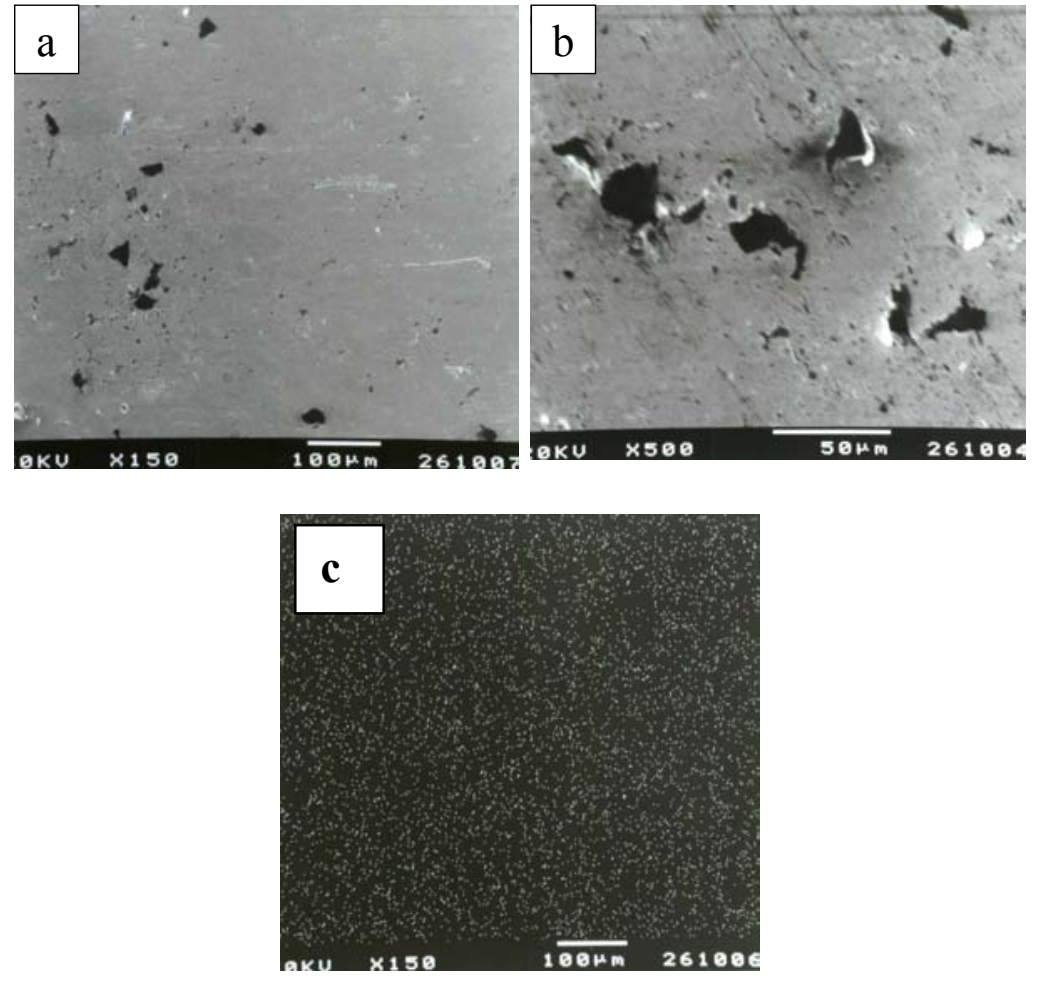

Figura 9. Imagens SEM e mapeamento de Hg obtidos para substrato de Ir posterior à remoção do $\mathrm{Hg}: \mathrm{C}_{\mathrm{Hg}(\mathrm{I})}=3.51 \times 10^{-6} \mathrm{~mol} \mathrm{~L}^{-1}$ (eletrólito suporte: solução de $\mathrm{KNO}_{3}, \mathrm{C}=$ $1.00 \mathrm{~mol} \mathrm{~L}^{-1}, \mathrm{pH} 1.00\left(\mathrm{HNO}_{3}\right)$ ), energia do feixe: $30 \mathrm{kV}$. Ampliação: a) $150 \times$; b) $500 \times$; c) $150 \times$.

Com base nos resultados anteriores e para confirmar que não havia a influência da presença do $\mathrm{Hg}$ (I) proveniente da solução sobre o perfil IxE dos voltamogramas na remoção do $\mathrm{Hg}$, repetiu-se os experimentos, procedendo-se o depósito do $\mathrm{Hg}$ como descrito anteriormente, porém, trocando-se a matriz original por solução de eletrólito de suporte, para posterior oxidação do $\mathrm{Hg}$ previamente eletrodepositado. Tomou-se o cuidado, durante a troca de matriz, de lavar oito vezes a célula para eliminar o $\mathrm{Hg}(\mathrm{I})$ presente. Posterior à troca da matriz foi obtido o voltamograma para a redução do Hg, apresentado na Fig. 10. É possível verificar a partir desta figura que posterior à troca de matriz, o perfil 
da região anódica permanece inalterado, se comparado com a Fig. $7 b$ (condições experimentais idênticas). Comportamento desta natureza é indicativo de que a presença do $\operatorname{Hg}(\mathrm{I})$ em solução não exerce influência sobre o processo de dessorção do Hg depositado na condição de UPD.

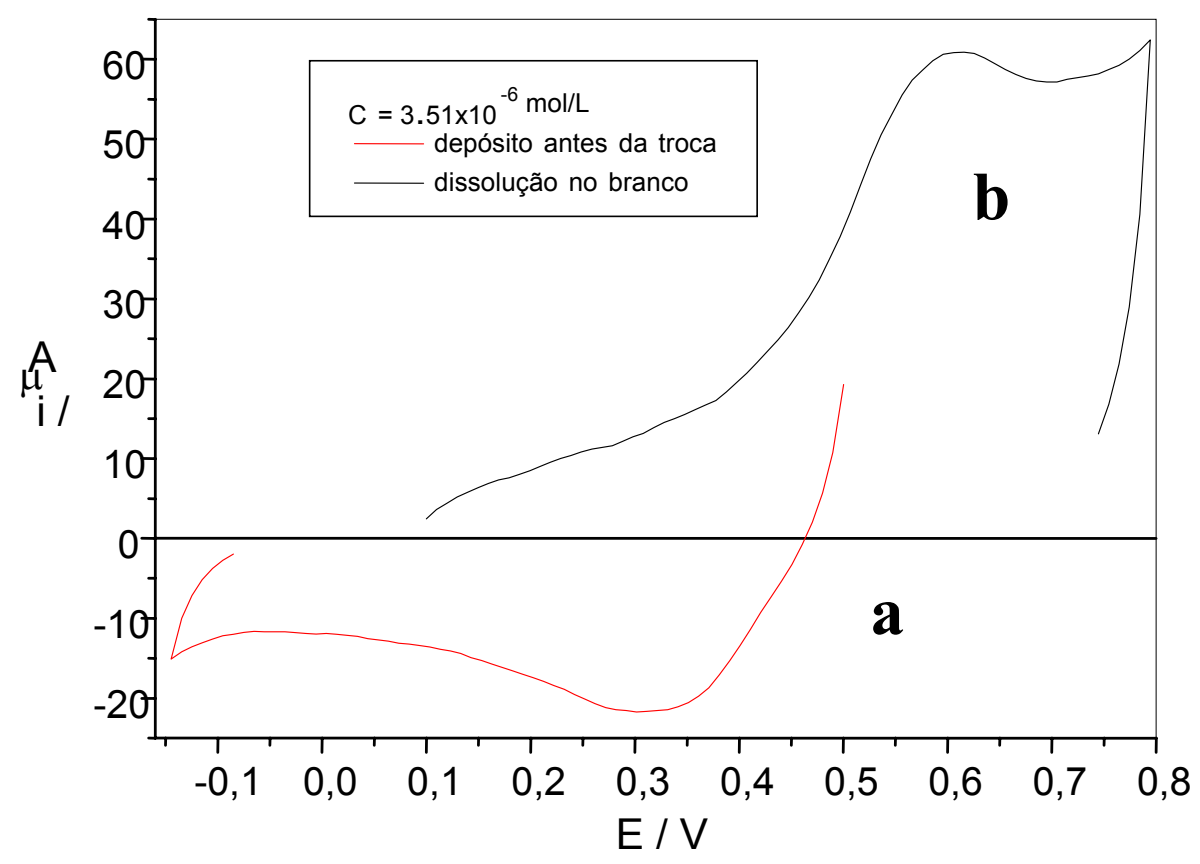

Figura 10. Voltamogramas cíclicos obtidos sobre o eletrodo de Ir puro no processo de troca de matriz: (a) após 20 min, com agitação constante sob fluxo de $\mathrm{N}_{2}$ na condição de circuito aberto, $\mathrm{C}_{\mathrm{Hg}(\mathrm{I})}=3.51 \times 10^{-6} \mathrm{~mol} \mathrm{~L}^{-1}, \mathrm{v}=50 \mathrm{mV} \mathrm{s}^{-1}$; (b) oxidação do $\mathrm{Hg}$ posterior a troca de matriz, $\mathrm{KNO}_{3} \mathrm{C}=1.00 \mathrm{~mol} \mathrm{l}^{-1}, \mathrm{pH} \mathrm{1.00.} \mathrm{T}=(25 \pm 1)^{\circ} \mathrm{C}$.

Entre o experimento de deposição e oxidação do $\mathrm{Hg}$ a amostra foi submetida à análise por microscopia eletrônica de varredura. As imagens SEM (Fig. 11) e os espectros de EDX, no entanto, não mostram a presença do $\mathrm{Hg}$. Considere-se que, posterior as análises de superfície foi observado uma carga igual a $607 \mu \mathrm{C} \mathrm{cm}^{-2}$, referente à remoção do $\mathrm{Hg}$ presente sobre o substrato. Na Fig. 11b observa-se a região de controle da lâmina (isolada com parafilm), sem contato com a solução contendo $\operatorname{Hg}(\mathrm{I})$. 

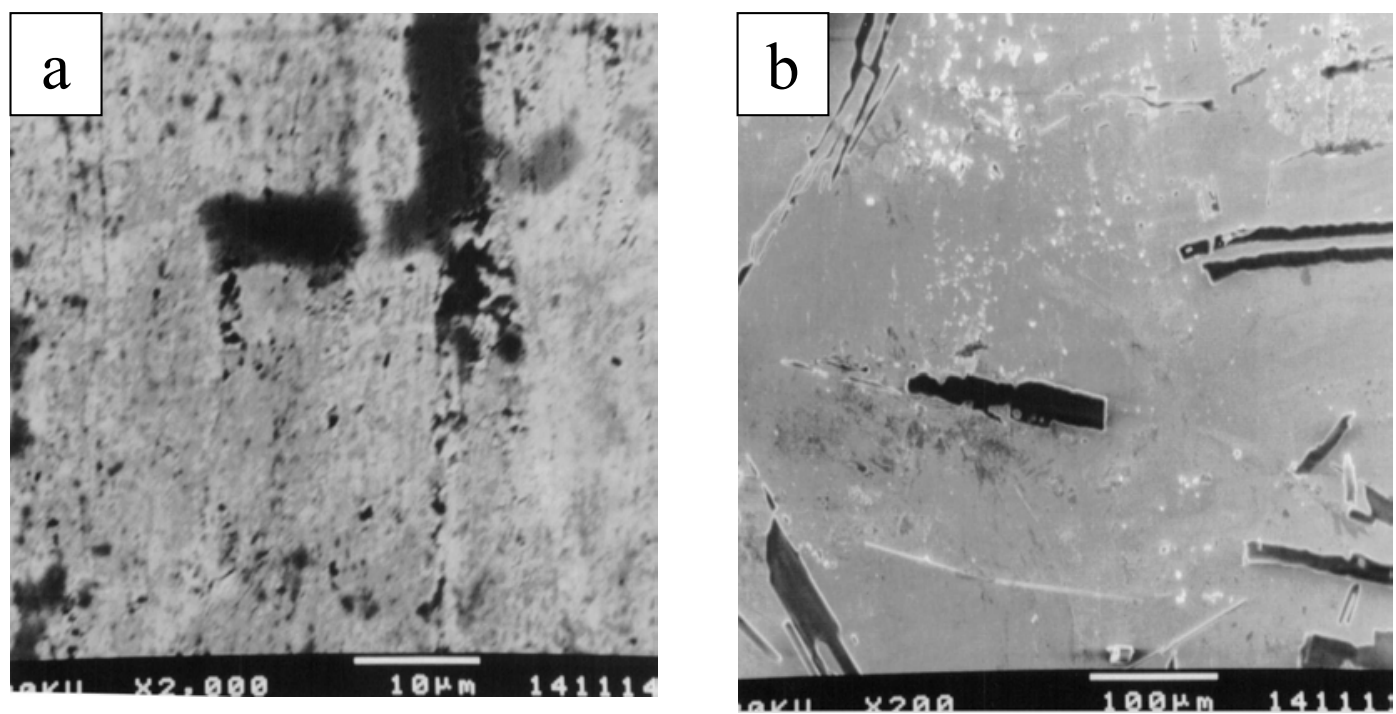

Figura 11. Imagens SEM obtidas para o substrato de $\mathrm{Ir}$ com $\mathrm{Hg}$ depositado na condição de UPD anterior à oxidação no eletrólito suporte: $\mathrm{C}_{\mathrm{Hg}(\mathrm{I})}=3.51 \times 10^{-6} \mathrm{~mol} \mathrm{~L}^{-1}$ (eletrólito de suporte: solução de $\left.\mathrm{KNO}_{3}, \mathrm{C}=1.00 \mathrm{~mol} \mathrm{~L}^{-1}, \mathrm{pH} 1.00\left(\mathrm{HNO}_{3}\right)\right)$, energia do feixe: 30 $\mathrm{kV}$ : a) região de contato com $\mathrm{Hg}(\mathrm{I})$ : ampliação $=2000 \times$; b) região de controle: ampliação $=200 \times$.

\section{Conclusões}

Os resultados permitem concluir que, independente do tratamento inicial, observa-se a presença constante de um filme fino de óxido constantemente sobre o substrato, cuja formação não está relacionada necessariamente ao avanço do potencial para a região de descarga de $\mathrm{O}_{2}$, ocorrendo concomitantemente com a deposição / remoção do filme de $\mathrm{Hg}$.

A deposição de $\mathrm{Hg}$ ocorre na condição de circuito aberto, impossibilitando trabalhar com eletrodos de $\mathrm{Ir}$ em meio contendo o $\mathrm{Hg}$ sem que haja a contaminação do substrato e, consequentemente, mudanças no perfil dos voltamogramas obtidos.

A obtenção do filme fino de $\mathrm{Hg}$ na condição de UPD é possível para $3.51 \times 10^{-7}$ $\leq \mathrm{C}_{\mathrm{Hg}(\mathrm{I})} \leq 3.51 \times 10^{-6} \mathrm{~mol} \mathrm{~L}^{-1}$.

\section{Agradecimentos}

Os autores agradecem à FAPESP (Fundação de Amparo à Pesquisa do Estado de São Paulo) e ao CNPq/PIBIC (Conselho Nacional de Desenvolvimento Científico e Tecnológico / Programa Institucional de Bolsas de Iniciação Científica) pelo apoio financeiro a este trabalho. 


\section{Referências}

1. I.C. Hwang and S.I. Woo, Zeolites and Related Micropor 84 (1994) 757.

2. P. Albers, B. Engler, J. Leyner, E. Lox, G. Prescher and K.Seibold, Chem. Eng. Technol. 17 (1994) 161.

3. G.E. Poirier, B.K. Hance and J.M. White, J. Phys.-Chem. 97 (1993) 5965.

4. A. Talo, J. Lahtinen, P. Hautojarvi, Appl. Catal. B-Environ. 5 (1995) 221.

5. A. Aramata and M. Masuda, J. Electrochem. Soc. 138 (1991) 1949.

6. R.W. Joyner and E.S. Shpiro, Catalysis Letter 9 (1991) 239.

7. E. Xue, K. Seshan, J.G. Vanommem and J.R.H. Ross, Appl. Caltal. BEnviron. 2 (1993) 183.

8. J.L.G. Fierro, J.M. Palacios and F.Tomas, J. Mater. Sci. 27 (1992) 685.

9. K.G. Kreider, M.J. Tarlov and J.P. Cline, Sensor Actuator B-Chem. 289 (1995) 165 .

10. E. Bolzán, M.I. Florit and A.J. Arvia, J. Electroanal. Chem. 461 (1999) 40.

11. E. Martins, R.C. Salvarezza and A.J. Arvia, Electrochem. Acta 43 (1998) 549.

12. E. Martins, R.C. Salvarezza and A.J. Arvia, Electrochem. Acta 41 (1996) 2441.

13. C. Chialvo, W.E. Triaca and A.J. Arvia, J. Electroanal. Chem. 146 (1983) 93.

14. F.L. Fertonani, E. Milaré, M. Ionashiro, A.V. Benedetti, J. Thermal Analysis and Calorimetry 67 (2002) 403.

15. F.L. Fertonani, A.V. Benedetti, M. Ionashiro, Thermochim. Acta 265 (1995) 151.

16. F.L. Fertonani, M. Yonashiro, P. Melnikov, F. Sanz and A.V. Benedetti. XII Congreso Iberoamericano, IX Encuentro Venezolano de Electroquimica - Livro de Resúmenes - 1996 (416).

17. F.L. Fertonani, Preparo e caracterização de eletrodos de mercúrio com substratos de platina, platina-irídio e platina-ródio (Tese de 
Doutoramento), 196 (1996).

18. E. Milaré, F.L. Fertonani, M. Ionashiro, A.V. Benedetti, P. Melnikov, XIII Congreso de la Sociedad Iberoamericana de Electroquimica - SIBAE 98, libro de resumenes. (1998) 140.

19. E. Milaré, F.L. Fertonani, M. Ionashiro, A.V. Benedetti, XI Simpósio Brasileiro de Eletroquímica e Eletroanalítica, Livro de Resumos - (1999) 419.

20. E. Milaré, F.L. Fertonani, M. Ionashiro, A.V. Benedetti, J. Thermal Anal. $59(2000) 617$.

21. E. Milaré, F.L. Fertonani, M. Ionashiro, A.V. Benedetti, $51^{a}$ Reunião Anual da SBPC, VI JNIC (Jornada Nacional de Iniciação Científica) (1999).

22. F.L. Fertonani, A.V. Benedetti, J. Servat, J. Portillo, F. Sanz, Thin Solid Films 341 (1999) 1.

23. M.C. Santos, S.A.S. Machado, L.A. Avaca, L.H. Mascaro, Química Nova $23(2000) 392$. 